\title{
Human IgM paraproteins demonstrate shared reactivity between Campylobacter jejuni lipopolysaccharides and human peripheral nerve disialylated gangliosides
}

\author{
Bart C. Jacobs ${ }^{\mathrm{a}, \mathrm{b}}$, Graham M. O’Hanlon ${ }^{\mathrm{c}}$, Elvera G. Breedland ${ }^{\mathrm{b}}$, Jean Veitch ${ }^{\mathrm{c}}$, \\ Pieter A. van Doorn ${ }^{a}$, Hugh J. Willison ${ }^{c, *}$ \\ ${ }^{a}$ Department of Neurology, Erasmus University, P.O. Box 1738, 3000 DR Rotterdam, The Netherlands \\ b Department of Immunology, Erasmus University, P.O. Box 1738, 3000 DR Rotterdam, The Netherlands \\ ${ }^{\mathrm{c}}$ University Department of Neurology, Institute of Neurological Sciences, Southern General Hospital, Glasgow, G51 4TF, UK
}

Received 18 April 1997; revised 16 June 1997; accepted 16 June 1997

\begin{abstract}
IgM paraproteins from patients with CANOMAD (chronic ataxic neuropathy, ophthalmoplegia, M-protein, agglutination, anti-disialosyl antibodies) react with $\operatorname{NeuAc}(\alpha 2-8)$ NeuAc epitopes on a wide range of gangliosides including GQ1b, GT1a, GD1b and GD3. The tissue distribution of reactive antigens in human peripheral nerve has not been addressed in detail. In addition, the origin of these antibodies is unknown. Here we report that purified anti-disialosyl paraproteins from two affected patients bind a wide array of human peripheral nerve structures including dorsal root ganglia, dorsal and ventral root axons, femoral and oculomotor nerves. We also show that these paraproteins bind lipopolysaccharides of Campylobacter jejuni isolates from $3 / 3$ cases of Miller Fisher syndrome, and to a less frequent extent, from cases of Guillain-Barré syndrome and enteritis controls. In conjunction with our previous studies, these data provide a possible causal link between the origin and pathogenic effects of anti-disialosyl antibodies in human paraproteinaemic neuropathy. (C) 1997 Elsevier Science B.V.
\end{abstract}

Keywords: Peripheral neuropathy; Miller Fisher syndrome; Campylobacter jejuni; Monoclonal gammopathy; Gangliosides; Molecular mimicry

\section{Introduction}

Phenotypic variants of autoimmune neuropathy are characterised by specific patterns of serum anti-ganglioside antibodies. One form of peripheral neuropathy associated with such an unique pattern of anti-ganglioside antibodies is an IgM paraproteinaemic neuropathy with core clinical features of chronic sensory ataxia and areflexia and with variably present ophthalmoplegia and/or red blood cell cold agglutinating (cold agglutinin) activity (Ilyas et al., 1985; Arai et al., 1992; Duane et al., 1992; Obi et al., 1992; Yuki et al., 1992; Willison et al., 1993a; Herron et al., 1994; Brindel et al., 1994). This has recently been reported under the acronym CANOMAD: chronic ataxic neuropathy with ophthalmoplegia, M-protein, agglutination

\footnotetext{
* Corresponding author. Tel.: +44-141-2012464; fax: +44-1412012993; e-mail: gora13@udcf.gla.ac.uk
}

and disialosyl antibodies (Willison et al., 1996). The IgM paraproteins found in patients with CANOMAD react with NeuAc $(\alpha 2-8) \mathrm{NeuAc}(\alpha 2-3) \mathrm{Gal}$ disialosyl epitopes present on a wide range of neural gangliosides including GQ1b, GT1a, GT1b, GD1b, GD3 and GD2. This or a structurally similar epitope is also present on red blood cell glycophorins, thereby accounting for the concurrent cold agglutinin disease due to red cell cold agglutinating activity of anti-Pr specificity in some CANOMAD cases (Roelcke and Kreft, 1984).

Some CANOMAD patients have fixed or intermittent episodes of ophthalmoplegia and/or bulbar dysfunction (Ilyas et al., 1985; Brindel et al., 1994, Herron et al., 1994) reminiscent of Miller Fisher syndrome (MFS), a variant of Guillain-Barré syndrome (GBS) characterised by acute ataxia, areflexia and ophthalmoplegia (Fisher, 1956). Over $90 \%$ of acute phase MFS sera also contain anti-GQ1b and anti-GT1a IgG antibodies (Chiba et al., 1992, 1993; Yuki et al., 1993; Willison et al., 1993b) which react with GD1b 
and GD3 in around half the cases (O'Leary et al., 1996). Thus, in addition to sharing similar clinical features, CANOMAD and MFS also share a similar pattern of serum anti-ganglioside antibodies. In CANOMAD the antibodies are of IgM class and chronically elevated, whereas in MFS which is an acute and self limiting disease, the antibodies are $\mathrm{IgG}$, subclass restricted to $\mathrm{IgG}_{1}$ and $\mathrm{IgG}_{3}$, and transiently present (Willison and Veitch, 1994).

The distribution of immunoreactive epitopes identified with CANOMAD sera in the human peripheral nervous system has not been thoroughly examined. In the mouse, we have shown that an IgM antibody cloned from an affected patient binds to many peripheral structures including dorsal root ganglion (DRG neurons), sensory and motor peripheral nerve trunks, motor nerve terminals and muscle spindles (Willison et al., 1996). One previous study using whole serum from a CANOMAD case has shown IgM binding to human DRG neurons but no staining was seen in other sites (Oka et al., 1996). In view of our studies in the mouse this seems surprising. Other studies have performed immunocytochemical localisation studies using murine monoclonal antibodies raised by ganglioside immunisation which have been selected for their high specificity for individual gangliosides including GQ1b (Chiba et al., 1993) and GD1b (Kusonoki et al., 1993). Although of interest in their own right, these antibodies are neither representative of the CANOMAD specificity, nor directly comparable with human autoantibodies. Here we report our findings that highly purified IgM paraproteins from CANOMAD cases have broader tissue immunoreactivity in man than previously recognised.

Campylobacter jejuni (C. jejuni) enteritis is a commonly recognised infection known to precipitate MFS and several investigators have isolated strains of $C$. jejuni from MFS subjects (Yuki et al., 1994; Jacobs et al., 1995; Salloway et al., 1996). Evidence indicates that anti-GQ1b ganglioside antibody responses in MFS are triggered as a result of molecular mimicry with structurally similar epitopes on lipopolysaccharides (LPS) from MFS-associated strains of $C$. jejuni. Serological studies using anti-GQ1b monoclonal antibodies and MFS sera have demonstrated GQ1b-like epitopes on whole organisms and LPS from strains of $C$ jejuni isolated from patients with MFS (Yuki et al., 1994; Jacobs et al., 1995, 1997). Structural characterisation of an MFS associated LPS core oligosaccharide has shown it to contain a terminal trisaccharide comprising $\operatorname{NeuAc}(\alpha 2-8) \operatorname{NeuAc}(\alpha 2-3) \mathrm{Gal}$ which is common to GQ1b, GT1a and GD3 gangliosides (Salloway et al., 1996). We therefore considered the possibility that the IgM paraproteins found in CANOMAD sera might also arise through antigenic stimulation by disialylated or polysialylated epitopes on core oligosaccharides of $C$. jejuni derived LPS. Here, we tested the reactivities of highly purified monoclonal IgM fractions containing anti-disialosyl antibodies from 2 CANOMAD patients with LPS from a panel of $C$. jejuni isolates from patients with MFS, GBS and controls.

\section{Materials and methods}

\subsection{Clinical subjects}

Clinical reports on patients VP (Herron et al., 1994)) and CH (Willison et al., 1993a, 1996) have been previously published. Both had features of a chronic, predominantly sensory neuropathy comprising peripheral sensory loss, profound limb ataxia, areflexia and mild or absent limb weakness. Interspersed on the chronic background in both patients (recognised subsequent to the 1993 report in patient $\mathrm{CH}$ ) was episodic deterioration with craniobulbar dysfunction, including ophthalmoplegia. Both patients had IgM lambda paraproteins with anti-Pr cold agglutinating activity and very high titre $\left(>1 / 10^{5}\right)$ anti-disialylated ganglioside antibodies, thereby comprising the CANOMAD phenotype. To assess CANOMAD sera for evidence of a recent $C$. jejuni infection, serum IgM, IgG, and $\operatorname{IgA}$ antibodies against a glycine extract of $C$. jejuni proteins were determined by ELISA, as previously described (Jacobs et al., 1996).

\subsection{Anti-ganglioside antibody assays and antibody purifi- cation}

Serum anti-ganglioside antibodies in CANOMAD patients $\mathrm{VP}, \mathrm{CH}$ and in neuropathy subjects $(\mathrm{A}-\mathrm{F})$ from whom $C$. jejuni strains were isolated were determined by enzyme linked immunosorbent assay (ELISA) and thin layer chromatography overlay (TLC overlay) using a panel of commercially available gangliosides as previously described (Willison et al., 1993a). IgM cold agglutinating antibodies from VP and $\mathrm{CH}$ were affinity purified from plasma by temperature-dependent elution on human group $\mathrm{O}$ red blood cells (RBC), as previously described (Willison et al., 1993a). These fractions were monitored by quantitative immunoassay for IgM and isoelectric focusing (IEF) with western blotting as previously described for $\mathrm{CH}$ and are referred to as rbc-M (Willison et al., 1996).

\subsection{Campylobacter jejuni isolates and lipopolysaccharide extraction}

The characteristics of the $C$. jejuni strains and subjects from whom they were derived are shown in Table 1. Organisms were isolated from stool cultures of 3 patients with MFS (A, B and C), 3 patients with GBS (D, E and F) and 3 enteritis patients without neurological involvement (1, 2 and 3), as described previously (Jacobs et al., 1997). Patient A, B and C had clinical features of ophthalmoplegia, ataxia and areflexia without limb weakness, and patients D, E and $\mathrm{F}$ fulfilled the diagnostic criteria for GBS. C. jejuni bacteria were grown on blood agar plates in a microaerobic atmosphere. After $48 \mathrm{~h}$ the bacteria were harvested, inactivated in $1 \%$ formaldehyde and washed in phosphate-buffered saline solution (PBS) ( $\mathrm{pH}$ 7.4). The 
Table 1

Serotypes of $C$. jejuni isolates and antibody profiles of neuropathy and enteritis control subjects from whom they were derived

\begin{tabular}{llll}
\hline Subject & Diagnosis & $\begin{array}{l}\text { Anti-ganglioside } \\
\text { antibody profile }\end{array}$ & $\begin{array}{l}\text { C. jejuni serotype } \\
\text { (Penner) }\end{array}$ \\
\hline A & MFS & GQ1b,GD1b, GD3 & O:4,50 \\
B & MFS & GQ1b & non-typable \\
C & MFS & GQ1b,GD1b, GD3 & O:23 \\
D & GBS & GM1, GD1b & O:1 \\
E & GBS & none detected & O:24 \\
F & GBS & not tested & non-typable \\
1 & enteritis & not tested & O:23 \\
2 & enteritis & not tested & O:22 \\
3 & enteritis & not tested & O:41 \\
\hline
\end{tabular}

LPS fractions from the $C$. jejuni bacteria were isolated by phenol-water extraction (Westphal and Jahn, 1965) and were demonstrated to contain less than $1 \%$ proteins. For control studies the LPS fractions were incubated with 0.05 $\mathrm{U} / \mathrm{ml}$ of sialidase from Clostridium perfringens or Arthrobacter ureafaciens (Sigma, St. Louis, MO) in 50 $\mathrm{mM}$ of sodium acetate buffer $(\mathrm{pH} 5.5)$ overnight at $37^{\circ} \mathrm{C}$. After incubation, the LPS were incubated at $80^{\circ} \mathrm{C}$ for 30 min to inactivate the sialidases.

\subsection{Detection of antibodies reactive with lipopolysaccha- rides}

IgM antibodies against LPS were tested by ELISA and TLC-overlay. ELISA was performed by coating 96-well polystyrene microtiter trays (Immuno Maxisorb, Nunc, Roskilde) with $1 \mu \mathrm{g}$ of LPS in $50 \mu \mathrm{l}$ PBS per well overnight. After the coating all steps were performed at $4^{\circ} \mathrm{C}$. Non-specific binding sites were blocked with PBS containing $1 \%$ bovine serum albumin (BSA) for $4 \mathrm{~h}$. After blocking, the plates were incubated with 0.1 to $10 \mathrm{mg} / \mathrm{ml}$ rbc-M from VP or CH in PBS-0.1\% BSA overnight. After washing with PBS, the plates were incubated with peroxidase-conjugated goat antihuman IgM (mu-chain specific) diluted 1:2500 in PBS-0.1\% BSA for $4 \mathrm{~h}$. After washing with PBS, the plates were developed with $O$-phenyl diamine (Sigma) in citrate buffer ( $\mathrm{pH}$ 5.0) and the optical densities were read at $490 \mathrm{~nm}$. TLC was performed on aluminium backed Kieselgel 60 WF254S high-performance TLC plates (Merck, Darmstadt) loaded with $7 \mu \mathrm{g}$ of LPS in methanol/water ( $4: 1$, by volume), and developed in $n$-propanol/water/25\% $\mathrm{NH}_{4} \mathrm{OH}$ (24:12:8, by volume). After chromatography, the plates were air-dried and dipped in a solution of $0.1 \%$ polyisobutylmethacrylate in $n$-hexane. The overlays and washings were performed at $4^{\circ} \mathrm{C}$. The plates were air-dried and blocked with PBS-1\% BSA for 1 $\mathrm{h}$ and incubated with 0.1 to $10 \mathrm{mg} / \mathrm{ml}$ of rbc-M in PBS- $0.1 \%$ BSA for $4 \mathrm{~h}$. After washing with PBS, the plates were incubated for $2 \mathrm{~h}$. with peroxidase-conjugated goat-anti-human IgM (mu-chain specific, Sigma) in PBS$0.1 \%$ BSA and washed with PBS. The plates were developed for 10-150 s using enhanced chemiluminescence (ECL, Amersham).

\subsection{Immunofluorescence studies}

Human spinal cord, dorsal root ganglia (DRG), dorsal and ventral roots, femoral nerve and 3rd cranial nerve were obtained from a middle aged male without neurological disease at post mortem subject to local ethical approval and guidelines. Tissues embedded in Tissue-Tek O.C.T. mounting medium (Miles Diagnostics, Elkhart, IN) were frozen in a slurry of ethanol and dry-ice and stored at $-70^{\circ} \mathrm{C}$. Cryostat sections $(5-15 \mathrm{~m})$ were cut onto 3aminopropyltriethoxysilane (APES) coated slides, and allowed to air-dry before immediate use or storage at $-20^{\circ} \mathrm{C}$. Mounted tissue sections were incubated with the rbc-M fractions from VP and $\mathrm{CH}$ at $40 \mu \mathrm{g} / \mathrm{ml}$, diluted in PBS containing $10 \%$ goat serum and $0.1 \%$ Triton X100 for $3 \mathrm{~h}$ at $4^{\circ} \mathrm{C}$. Mouse anti-neurofilament antibody (clone 1217, Affiniti Research Products, Exeter) was used at 1:1000 dilution. After primary antibody incubations, sections were drained, rinsed in four washes of cold PBS and incubated with rhodamine labelled goat anti-human IgM and fluorescein labelled goat anti-mouse $\mathrm{IgG}$ secondary antibodies (Southern Biotechnology) at 1:300 dilutions in staining medium for $1 \mathrm{~h}$ at $4^{\circ} \mathrm{C}$. The slides were again rinsed $\times 4$ in cold PBS before being mounted in Citifluor antifade (Citifluor, Canterbury), ringed with nail-varnish to minimise drying and stored at $4^{\circ} \mathrm{C}$ in the dark prior to viewing. Consistent fluorescence signals were totally dependent on strict maintenance of all solutions at $4^{\circ} \mathrm{C}$ at all times throughout the staining procedure.

Sections were also stained with the anti-neurofilament antibody plus an irrelevant human IgM monoclonal antibody and/or secondary anti-human IgM antibody to control for non-specific binding and fluorescence bleedthrough between detection channels: the threshold of the image acquisition equipment was calibrated such that the control level of staining was at or close to zero. Images were obtained by means of a Sony colour CCD camera mounted onto a Zeiss Axioplan fluorescent microscope and linked to a PC driven image archiving system (Sirrius VI; Optivision). Bitmap processing and annotation were conducted on PhotoMagic and Windows Draw (both by Micrographx). Images were printed directly using a photographic-quality colour printer (Kodak ColorEase).

\section{Results}

\subsection{Serological studies on CANOMAD subjects}

Patients VP and $\mathrm{CH}$ had anti-ganglioside IgM antibody titres previously reported as follows. For VP: GM1, < 100; GM2, 30,000; GM3, < 100; GD1a, 30,000; GD1b, 120,000; GT1b, 100,000; GQ1b, 170,000; GD3 150,000. For CH: GM1, < 100; GM2, < 100; GM3, 660; GD1a, 570; GD1b, 850,000; GT1b, 300,000; GQ1b, 450,000; GD3 260,000. The rbc-M fractions in both patients had an 


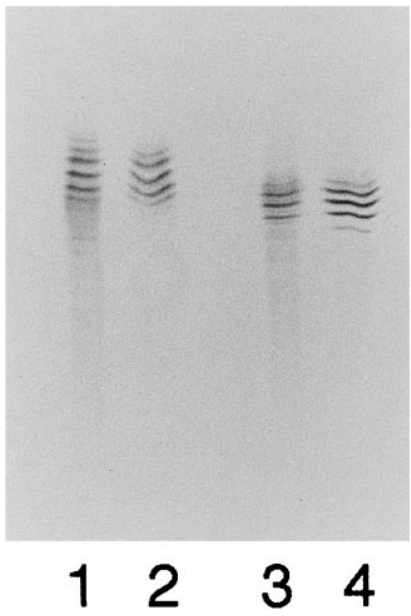

Fig. 1. Western blots of IEF gels of whole serum (lanes 1 and 3) and rbc-M (lanes 2 and 4) from VP (lanes 1 and 2) and $\mathrm{CH}$ (lanes 3 and 4) stained with anti-human IgM showing the monoclonal IgM on a background of polyclonal IgM in serum compared with the pure paraprotein of identical isoelectric mobility in rbc-M. Monoclonal immunoglobulins appear as ladders in IEF gels due to charge micro-heterogeneity.

identical pattern of anti-ganglioside antibody reactivity as the sera from which they were derived (data not shown). IEF western blotting of serum and rbc-M (Fig. 1) showed resolution of the rbc-M as a pure monoclonal ladder corresponding to the serum monoclonal IgM and free of polyclonal IgM when compared with the IEF profile of whole serum. No antibody reactivity against a $C$. jejuni glycine extract was detected in either whole serum or rbc-M from $\mathrm{VP}$ and $\mathrm{CH}$, indicating no recent $C$. jejuni infection.

\subsection{Reactivity of rbc-M fractions from $\mathrm{CH}$ and $\mathrm{VP}$ with $C$. jejuni lipopolysaccharides}

The rbc-M fractions from VP and $\mathrm{CH}$ bound with LPS from the $C$. jejuni isolates from all 3 MFS patients (subjects $\mathrm{A}, \mathrm{B}$ and $\mathrm{C}$ ), and from one GBS patient (subject $\mathrm{E}$ ) and one enteritis control (subject 3) as shown in Fig. 2. These patterns of binding were confirmed in TLC-overlay in which LPS from MFS A, B and C, GBS E and enteritis control 3 were positive and LPS from GBS D and F and enteritis controls 1 and 2 were negative (Fig. 3). Binding of rbc-M from VP and $\mathrm{CH}$ to LPS was found to be higher at $4^{\circ} \mathrm{C}$ than at $37^{\circ} \mathrm{C}$ incubation as was also previously seen for ganglioside binding (data not shown). The capacity of the LPS to bind rbc-M from both VP and $\mathrm{CH}$ decreased after treatment of the LPS with sialidases from Clostridium perfringens (Fig. 2) and Arthrobacter ureafaciens (data not shown). No activity was demonstrated against LPS from the $C$. jejuni isolates of GBS subjects D and F and control subjects 1 and 2 . There was no obligate association between the presence of the paraprotein binding epitopes on LPS and the Penner serotype of the bacteria: enteritis control 1 is Penner O:23 and does not bind the paraproteins, although it has the same O:23

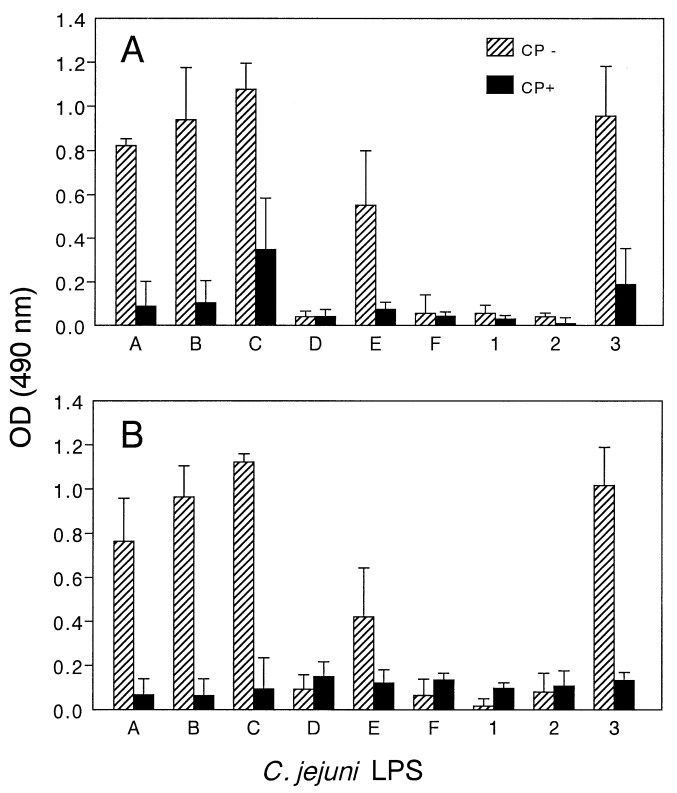

Fig. 2. ELISA analysis of the reactivity of rbc-M from patients VP (1 $\mu \mathrm{g} / \mathrm{ml}$; panel A) and $\mathrm{CH}(10 \mu \mathrm{g} / \mathrm{ml}$; panel B) with LPS from $C$. jejuni isolates without (hatched bars) and with (solid bars) treatment with Clostridium perfringens neuraminidase. LPS from all 3 MFS-associated C. jejuni strains (lanes A, B and C) are reactive compared with LPS from one of the GBS-associated C. jejuni strains (lanes D, E and F) and one enteritis control strain (lanes 1, 2 and 3). Means + 2SD.

serotype as the isolates from MFS patient $\mathrm{C}$, which does bind the paraproteins.

\subsection{Immunofluorescence studies on human peripheral nerve}

The binding patterns of rbc-M from $\mathrm{VP}$ and $\mathrm{CH}$ to a wide range of human peripheral nerve structures were identical. In spinal cord, the rbc-M bound amorphously to grey matter including weak cytoplasmic labelling of mo-

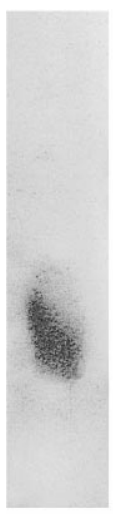

A

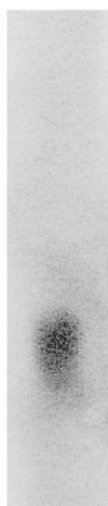

B

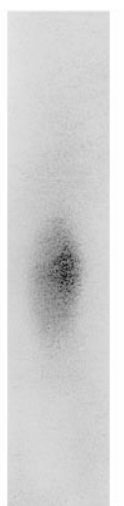

C

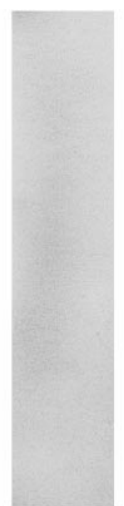

D

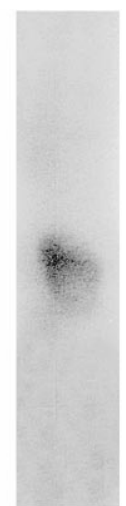

E
Fig. 3. TLC-overlay analysis of LPS ( $7 \mu \mathrm{g} /$ lane) from 3 MFS-associated C. jejuni strains (panels A, B and C) and 2 of the GBS-associated $C$. jejuni strains (panels $\mathrm{D}$ and $\mathrm{E}$ ) immunostained with rbc-M from patient $\mathrm{CH}(10 \mu \mathrm{g} / \mathrm{ml})$. Reactivity patterns correspond to those seen with ELISA. 
torneuron cell bodies. There was very faint binding to white matter tracts. In the DRG (Fig. 4) the majority of neuronal cell bodies displayed granular staining of an area including, and extending slightly beyond the neurofilament-positive region (Fig. 4, panels a and b), although a small population were unstained. In some areas of the DRG there was also weaker staining of extracellular regions surrounding the cell bodies. In fibre bundles within the DRG, there was strong staining associated with the axons of large fibres (Fig. 4, panels c and d), little or no staining of the myelin sheath, and surrounding haloes of staining in the extra cellular space (Fig. 4, panels e and f). Strong labelling of blood vessel walls was seen throughout the nervous system, here shown in the DRG (Fig. 4, panel $\mathrm{d}$, arrow). In the spinal roots (Fig. 5, panels a and b), both the dorsal and ventral root large fibre axons and surrounding extracellular regions were similarly stained, with little labelling evident in their compact myelin sheaths. A similar pattern was observed in the 3rd cranial nerve (Fig. 5, panels $\mathrm{c}$ and $\mathrm{d}$ ). Large fibres in the femoral nerve were
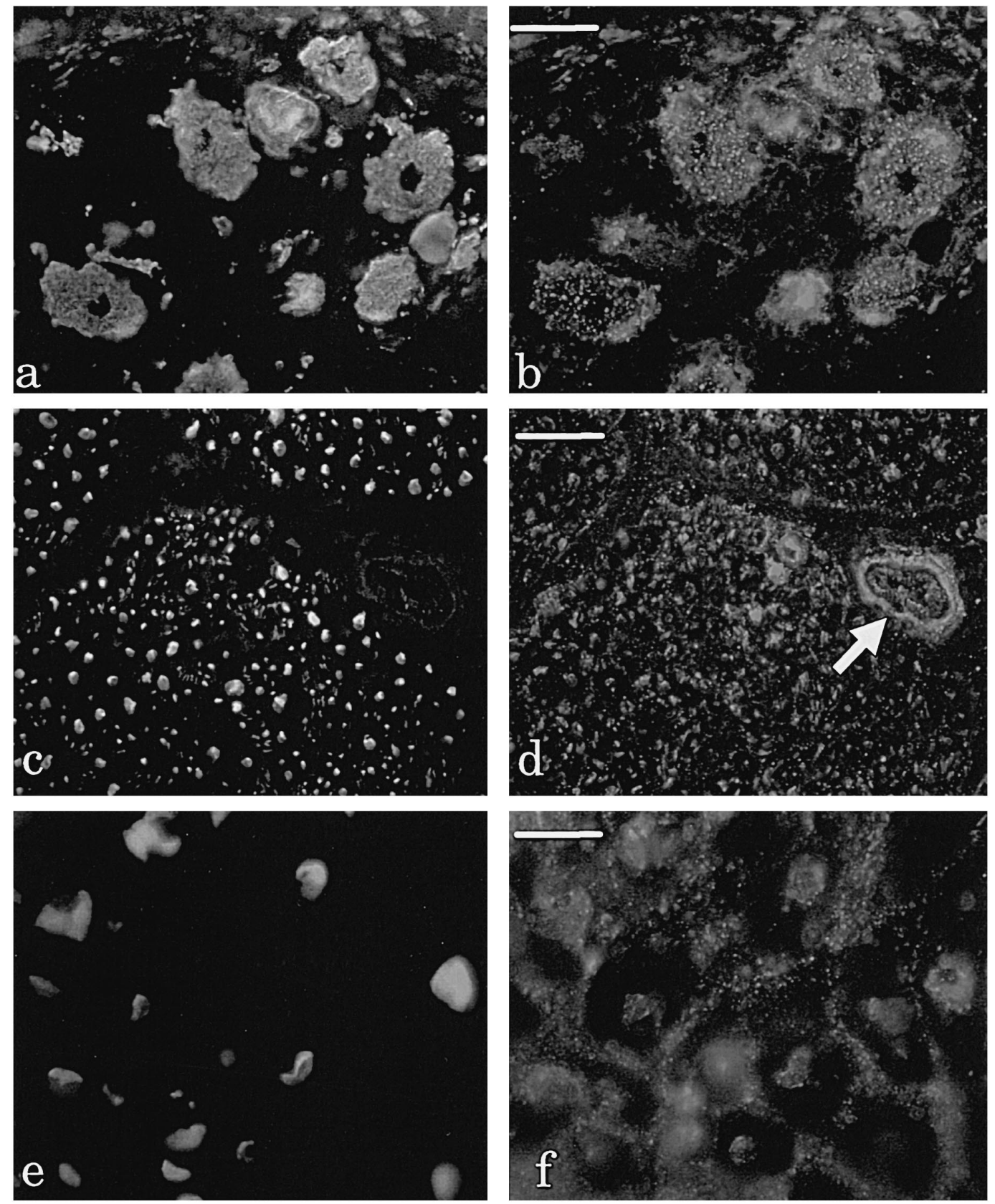

Fig. 4. Double-labelled pairs of fluorescent micrographs showing the immunolocalisation of rbc-M (panels b, $\mathrm{d}$ and $\mathrm{f}$ ) and anti-neurofilament antibodies (panels a, $\mathrm{c}$ and e) in human dorsal root ganglion. The majority of cell bodies are labelled (panels a and b; bar $=50 \mu \mathrm{m}$ ). In fibre bundles within the DRG staining was localised to axonal profiles, connective tissue and blood vessels (arrow; panels $\mathrm{c}$ and $\mathrm{d}$; bar $=50 \mu \mathrm{m}$ ). At higher magnification, labelling of large axons and the extracellular regions surrounding their myelin sheaths is seen (panels e and $\mathrm{f}$; bar $=20 \mu \mathrm{m}$ ). 
also stained in this pattern. Longitudinal sections confirmed this pattern and did not show significant compact or paranodal myelin staining. Small diameter fibre bundles in the femoral nerve, identifiable by clusters of neurofilament positive profiles were strongly immunoreactive (Fig. 5, panels e and f) although it was not possible to identify specific structures within the bundles at this resolution. These small fibre bundles were also readily identifiable within the dorsal root but were absent (as expected from the fibre size distribution) in the ventral root.

\section{Discussion}

In these studies we have shown that $\operatorname{IgM}$ paraproteins from patients with CANOMAD share immunoreactive determinants with human peripheral nerve antigens and $C$. jejuni LPS derived from MFS patients. These data provide a possible pathogenic link between the origin and the effect of anti-disialylated ganglioside $\operatorname{IgM}$ paraproteins in humans. They and also add further support to the view that CANOMAD and MFS are related polyneuropathies, char-
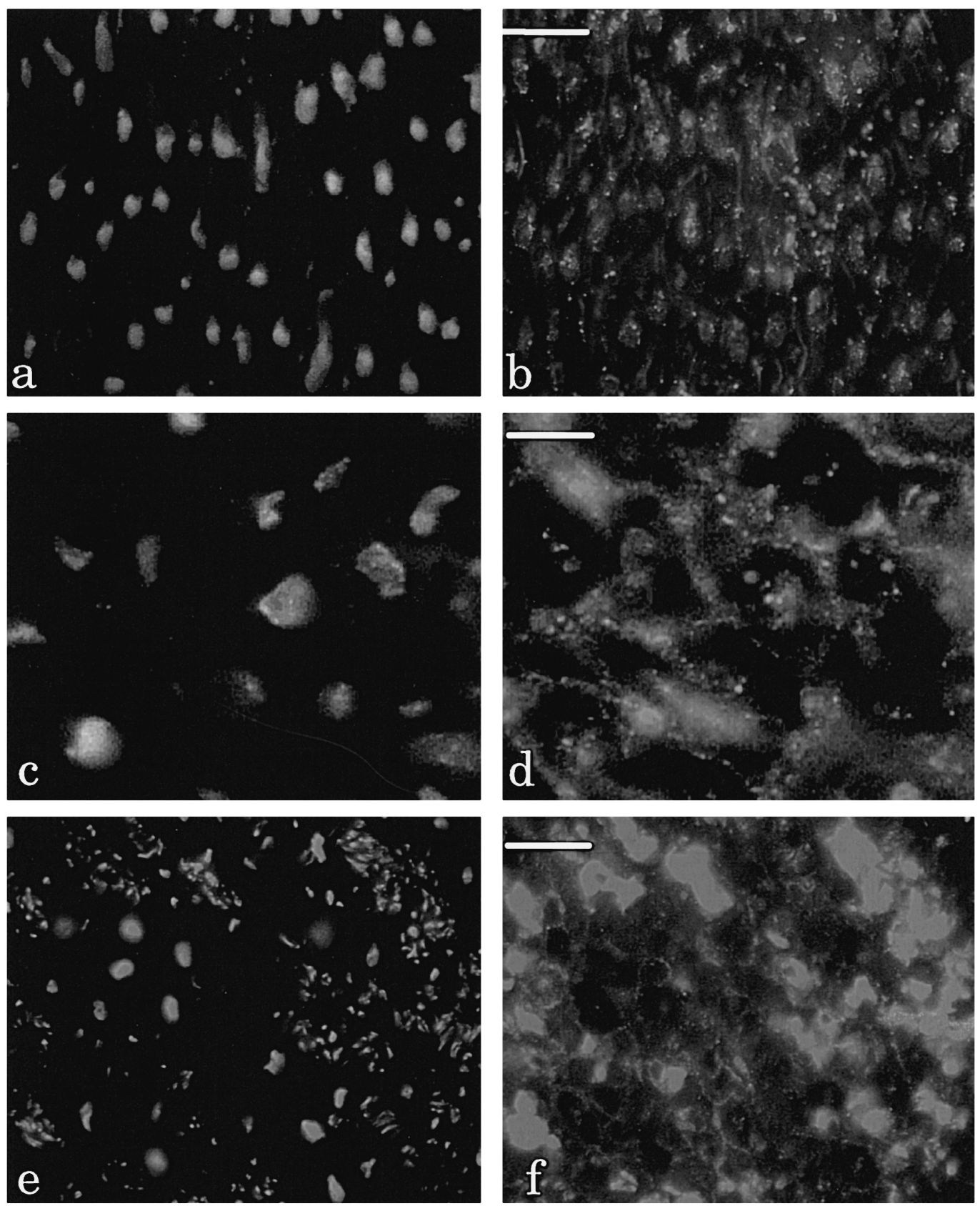

Fig. 5. Double-labelled pairs of fluorescent micrographs showing the immunolocalisation of rbc-M (panels $b$, $d$ and $f$ ) and anti-neurofilament antibodies (panels a, c and e) in human peripheral nerve. Axonal profiles and extracellular regions are stained in the ventral root (panels a and $\mathrm{b}$; bar $=20 \mu \mathrm{m}$ ) and 3rd cranial nerve (panels $\mathrm{c}$ and $\mathrm{d}$; bar $=10 \mu \mathrm{m}$ ). In the femoral nerve staining is intense over small fibre bundles (panels e and $\mathrm{f}$; bar $=20 \mu \mathrm{m}$ ). 
acterized by anti-ganglioside antibodies with overlapping but distinct specificity.

All MFS sera that bind GQ1b also bind GT1a and 50\% of these MFS sera also bind other gangliosides bearing at least one NeuNAc $(\alpha 2-8)$ NeuNAc- residue, such as GD1b and GD3 (Willison et al., 1994). The specificity of CANOMAD paraproteins for gangliosides is broader in that both the paraproteins described here bind to all $\mathrm{Ne}$ uNAc $(\alpha 2-8)$ NeuNAc- bearing gangliosides we have studied and may also bind very weakly to other gangliosides including GD1a and GM2 (Willison et al., 1993a; Herron et al., 1994). Anti-GQ1b antibodies in sera from MFS patients and murine monoclonal anti-GQ1b antibodies react with $C$. jejuni LPS derived from MFS cases, indicating that these LPS molecules bear a GQ1b-like epitope (Yuki et al., 1994; Jacobs et al., 1995). Interestingly the LPS core oligosaccharide from the only MFS associated $C$. jejuni to be structurally chacterised to date bears a GD3-like terminal trisaccharide rather than a GQ1b or GT1a motif (Salloway et al., 1996). In addition, Aspinall et al. (1994) have reported the structure of a GBS-associated LPS as containing a GT1a-like motif. It would thus appear that there may not be an exact relationship between the LPS oligosaccharide structure and the fine specificity of the corresponding anti-ganglioside antibody serology. Our data show that CANOMAD paraproteins not only react with LPS from all 3 MFS-associated C. jejuni strains but also bound $1 / 3$ GBS strains and 1/3 control strains, which were not bound by MFS sera (Jacobs et al., 1997). This indicates that CANOMAD paraproteins have a broader specificity for sialidase sensitive LPS structures than MFS sera and thus may arise through an immune response to a wider range of disialylated LPS structures than MFS associated antibodies.

There is no direct evidence that the paraproteins in CANOMAD have arisen in response to $C$. jejuni LPS. Neither of the CANOMAD cases reported enteritis as an initiating event at the onset of neurological symptoms as is seen in MFS. Equally, neither of the CANOMAD sera contained any detectable antibodies to $C$. jejuni protein antigens as assessed by routine $C$. jejuni serology: this indicates that any infection is currently inactive and any putative infection must have occurred in the distant past such that serum antibody levels have now decayed to sub-detectable levels. Our V region sequencing studies on the paraprotein which we have cloned from patient $\mathrm{CH}$ have shown that the antibody is encoded by immunoglobulin variable region genes with extensive somatic mutation; this is indicative of an origin through antigen driven maturation rather than dysregulated proliferation of a naive B-cell with a random antigen specificity as the primary event (Willison et al., 1996). The appearance of paraproteins in CANOMAD would be consistent with an antigen specific response to bacterial LPS, possibly chronically sequestered in gut associated lymphoid tissue and possibly arising from $C$. jejuni or other infections with cross reac- tive motifs. Assuming that MFS-associated anti-GQ1b antibodies and CANOMAD paraproteins arise in response to C. jejuni LPS, the major difference is that the former comprises an acute, self limiting primary immune response with class switching from IgM to higher affinity IgG antibodies whereas the latter comprises a chronic accumulation of IgM produced by a slowly expanding dysregulated clone of B cells.

We have already demonstrated in the MF1 mouse that monoclonal CANOMAD paraproteins can bind widely to murine peripheral nerve structures including dorsal root ganglia, motor nerve terminals, peripheral nerve axons and myelin and nodes of Ranvier. In addition, the paraprotein can immunolocalise to and induce electrophysiological abnormalities in motor nerve terminals in the ex vivo hemi-diaphragm model of nerve transmission, selected for study as one site out of many in the peripheral nerve unit that could be pathologically affected in the human disease (Willison et al., 1996). In our immunolocalisation studies in the human peripheral nervous system described here we have shown widespread binding to sensory and motor nerves, including the 3rd cranial nerve, with prominent staining in groups of dorsal root ganglion neurons. In view of the sensory ataxia seen in CANOMAD cases, the dorsal root ganglion, a site with a limited blood nerve barrier into which antibody can easily penetrate, seems a likely target for immunopathological attack. We have also observed extensive extracellular binding which would be unlikely to represent binding to gangliosides in this site but may represent binding to sialylated extracellular matrix components. The pattern of binding to human DRG neurons we have seen with CANOMAD monoclonal paraproteins is similar to that seen in previous reports using anti-GD1b antibodies. Using a murine anti-GD1b monoclonal antibody, Kusonoki et al. (1993) have immunolocalised GD1b to dorsal root ganglion neuronal cytoplasm and spinal root axons. In studies by Oka et al. (1996), using an unpurified CANOMAD serum of very similar specificity to those used here, a granular pattern of binding was seen in the DRG neuronal cell bodies, again very similar to that seen in this study. However, they observed no staining elsewhere in peripheral nerve in marked contrast to our CANOMAD IgM antibodies which stain human dorsal and ventral roots and peripheral nerve axons extensively. Thus, assuming that technical factors have not created the difference between our study and that of Oka and colleagues, there must be critical differences in antibody fine specificity which dictate immunoreactivity in different peripheral nerve regions. These may be reflected by clinical differences between patients.

Combined data from this and our previous studies suggest the following pathophysiological sequence of events in the evolution of CANOMAD: (a) individuals are infected with $C$. jejuni or other pathogens bearing disialylated ganglioside-like epitopes, (b) a low level, low affinity IgM response is mounted with insufficient autopathogenic 
potential to cause an acute post-infectious neuropathy, (c) transformation from a state of antigen dependent B cells proliferation to autonomous $\mathrm{B}$ cell proliferation occurs, with the formation of a monoclonal IgM gammopathy, (d) serum IgM antibody levels rise to a level at which binding kinetics favour sufficient antibody 'on' time to activate pro-inflammatory pathways in target membranes of the DRG and other antigen rich sites.

\section{Acknowledgements}

We are very grateful to Dr. L.S. Illis for his permission to study serum from patient VP. This work was supported by grants to Dr. Jacobs from the Prinses Beatrix Fonds (no. 90-3161 and no. 95-0519) and the Willem H. Kröger Stichting (no. 92-011) and grants to Dr. Willison from the Scottish Motor Neurone Disease Association and Guillain-Barré Support Group of Great Britain. Dr. Willison is a Wellcome Trust Senior Research Fellow in Clinical Science.

\section{References}

Arai, M., Yoshino, H., Kusano, Y., Yasaki, Y., Ohnishi, Y., Miyatake, T., 1992. Ataxic polyneuropathy and anti- $\operatorname{Pr}_{2} \operatorname{IgM} \kappa$ M proteinaemia. J. Neurol. 239, 147-151.

Aspinall, G.O., McDonald, A.G., Pang, H., Kurjanczyk, L.A., Penner, J.L., 1994. Lipopolysaccharides from Campylobacter jejuni serotype O:19. Structures of the core oligosaccharide regions from the serostrain and two bacterial isolates from patients with the Guillain-Barré syndrome. Biochemistry 33, 241-249.

Brindel, I., Preud'Homme, J., Vallat, J., Vincent, D., Vasquez, J., Jauberteau, M., 1994. Monoclonal IgM reactive with several gangliosides in a chronic relapsing neuropathy. Neurosci. Lett. 181, 103-106.

Chiba, A., Kusonoki, S., Shimizu, T., Kanazawa, I., 1992. Serum IgG antibody to ganglioside GQ1b is a possible marker of Miller Fisher syndrome. Ann. Neurol. 31, 677-679.

Chiba, A., Kusunoki, S., Obata, H., Machinami, R., Kanazawa, I., 1993. Serum anti-GQ1b IgG antibody is associated with ophthalmoplegia in Miller Fisher syndrome and Guillain-Barré syndrome. Clinical and immunohistochemical studies . Neurology 43, 1911-1917.

Duane, G.C., Farrer, R.G., Dalakas, M.C., Quarles, R.H., 1992. Sensory neuropathy with monoclonal immunoglobulin $\mathrm{M}$ to GD1b ganglioside. Ann. Neurol. 31, 683-685.

Herron, B., Willison, H.J., Veitch, J., Roelcke, D., Illis, L.S., Boulton, F.E., 1994. Monoclonal IgM cold agglutinins with anti-Pr1d specificity in a patient with peripheral neuropathy. Vox. Sang. 67, 58-63.

Ilyas, A.A., Quarles, R.H., Dalakas, M.C., Fishman, P.H., Brady, R.O., 1985. Monoclonal IgM in a patient with paraproteineamic polyneuropathy binds to gangliosides containing disialosyl groups. Ann. Neurol. 18, 655-659.
Jacobs, B.C., Endtz, H., van der Meche, F.G.A., Hazenberg, M.P., Achtereekte, H.A., Van Doorn, P.A., 1995. Serum anti-GQ1b antibodies recognize surface epitopes on Campylobacter jejuni from patients with Miller Fisher syndrome. Ann. Neurol. 37, 260-264.

Jacobs, B.C., Hazenberg, M.P., van Doorn, P.A., Endtz, H.Ph., van der Meche, F.G.A., 1997. Cross-reactive antibodies against gangliosides and Campylobacter jejuni lipopolysaccharides in patients with Guillain-Barré syndrome and Miller Fisher syndrome. J. Infect. Dis. 175, 729-734.

Kusonoki, S., Chiba, A., Tai, T., Kanazawa, I., 1993. Localisation of GM1 and GD1b antigens in the human peripheral nervous system. Muscle Nerve 16, 752-756.

Obi, T., Kusunoki, S., Takatsu, M., Mizoguchi, K., Nishimura, Y., 1992. IgM M-protein in a patient with sensory-dominant neuropathy binds preferentially to polysialogangliosides. Acta Neurol. Scand. 86, 215218

Oka, N., Kusaka, H., Kusonoki, S., Tsuda, H., Raji, R., Imai, T., Akiguchi, I., Kimura, J., 1996. IgM M-protein with antibody activity against gangliosides with disialosyl residue in sensory neuropathy binds to sensory neurons. Muscle Nerve 19, 528-530.

O'Leary, C.P., Veitch, J., Durward, W.F., Thomas, A.M., Rees, J.H., Willison, H.J., 1996. Acute oropharyngeal palsy is associated with antibodies to GQ1b and GT1a gangliosides. J. Neurol. Neurosurg. Psychiatr. 61, 649-652.

Roelcke, D., Kreft, H., 1984. Characterisation of various anti-Pr cold agglutinins. Transfusion 24, 210-213.

Salloway, S., Mermel, L.A., Seamans, M., Aspinall, G.O., Nam Shin, J.E., Kurjanczyk, L.A., Penner, J.L., 1996. Miller Fisher syndrome associated with Campylobacter jejuni bearing lipopolysaccharide molecules that mimic human ganglioside GD3. Infect. Immun. 64, 2945-2949.

Willison, H.J., Paterson, G., Veitch, J., Inglis, G., Barnett, S.C., 1993a. Peripheral neuropathy associated with monoclonal $\mathrm{IgM}$ anti-Pr${ }_{2}$ cold agglutinins. J. Neurol. Neurosurg. Psychiatr. 56, 1178-1184.

Willison, H.J., Veitch, J., Paterson, G., Kennedy, P.G.E., 1993b. Miller Fisher syndrome is associated with serum antibodies to GQ1b ganglioside. J. Neurol. Neurosurg. Psychiatr. 56, 204-206.

Willison, H.J., Veitch, J., 1994. Immunoglobulin subclass distribution and binding characteristics of anti-GQ1b antibodies in Miller Fisher syndrome. J. Neuroimmunol. 50, 159-165.

Willison, H.J., O'Hanlon, G.M., Paterson, G.J., Veitch, J., Wilson, G., Roberts, M., Tang, T., Vincent, A., 1996. A somatically mutated human anti-ganglioside antibody that induces experimental neuropathy in mice is encoded by the variable region heavy chain gene, V1-18. J. Clin. Invest. 97, 1155-1164.

Yuki, N., Miyatani, N., Sato, S., Hirabayashi, Y., Yamazaki, M., Yoshimura, N., Hayashi, Y., Miyatake, T., 1992. Acute relapsing sensory neuropathy associated with IgM antibody against B-series gangliosides containing a GalNAc $\beta 1-4(\mathrm{Gal} 3-2 \alpha$ NeuAc8$2 \alpha$ NeuAc) $\beta 1$ configuration. Neurology 42, 686-689.

Yuki, N., Sato, S., Tsuji, S., Ohsawa, T., Miyatake, T., 1993. Frequent presence of anti-GQ1b antibody in Fisher's syndrome. Neurology 43, 414-417.

Yuki, N., Taki, T., Takahashi, M., Saito, K., Yoshino, H., Tai, T., Handa, S., Miyatake, T., 1994. Molecular mimicry between GQ1b ganglioside and liposaccharides of Campylobacter jejuni isolated from patients with Fisher's syndrome. Ann. Neurol. 36, 791-793. 